\title{
Editorial Column
}

\section{Health Services Research: Critical Measurement Issues}

As the largest integrated health care system in the United States, the Veterans Health Administration (VHA) is responsible for treating more than 5 million patients each year at 1,400 sites of care that include medical centers, hospitals, clinics, and nursing homes. In addition, with an emphasis on care coordination and the advances in telehealth, VHA is able to provide some veterans with specialized care in the privacy of their own homes. Over the last decade, the number of patients served annually has more than doubled and encompasses an increasing number of elderly, chronically ill, and female veterans. VHA is currently preparing to care for increasing numbers of soldiers from Operation Iraqi Freedom and Operation Enduring Freedom, as they transition from active service to veteran. Despite these unprecedented demands on its resources, VHA continues to provide high quality care to our nation's veterans.

However, maintaining this course and level of performance is a major challenge, given the increasing numbers of veterans and the on-going fiscal constraints experienced by the VHA. That said, it is clear that measuring performance and outcomes is essential for continuous quality improvement efforts. To help in this arena, the Veterans Administration Health Services Research and Development (VA HSR\&D) Service established the Measurement Excellence and Training Resource Information Center (METRIC), which is dedicated to serving the measurement needs of VA researchers in every phase of research.

METRICs mission is to assist researchers in their search for measurement instruments that best fit individual research situations, to forge links between VA researchers and national measurement experts, to educate researchers on all aspects of the measurement process, and to contribute to the overall advancement of measurement science for VA and the broader healthcare community. METRIC investigators have contributed to this special issue of Health Services Research that focuses on measurement issues in health services research and on the potential contributions of measurement 
toward quality improvement efforts. The Special Issue covers an array of vital topics including discussion of how the validity and reliability of measurements can be affected by setting-specific and population-specific considerations. Articles in this issue also review alternative measurement methodologies, such as the use of proxies and other external-raters, as well as dynamic assessment of health.

HSR\&D is committed to the pursuit of health care quality through the acquisition of new health services knowledge and the dissemination, translation and implementation of that knowledge into improved patient health and care. METRIC plays a key role in this mission, and this comprehensive special issue provides important information on measurement issues that apply not only to VHA but to the broader healthcare community.

Shirley Meehan Ph.D., MBA Acting Director, VA HSR\&D 